\title{
ANTIMICROBIAL INVESTIGATION AND BINDING MODE ANALYSIS OF SOME NEWLY SYNTHESIZED 4-AMINO-5-((ARYL SUBSTITUTED)-4H-1, 2, 4-TRIAZOLE-3-YL)-THIO LINKED HYDROXAMIC ACID DERIVATIVES
}

\author{
MOUSUMI DAS ${ }^{1}$, BHASKAR DAS ${ }^{1}$, ARNAB DE ${ }^{1}$, SUBHASIS BANERJEE ${ }^{2}$, AMALESH SAMANTA ${ }^{1 *}$ \\ ${ }^{1}$ Department of Pharmaceutical Technology, Division of Microbiology and Biotechnology, Jadavpur University, Kolkata, West Bengal, India. \\ ${ }^{2}$ Department of Pharmaceutical Chemistry, Gupta College of Technological Sciences, Ashram More, Asansol, Kolkata - 713 301, \\ West Bengal, India. Email: asamanta61@yahoo.co.in \\ Received: 22 November 2018, Revised and Accepted: 04 January 2018
}

ABSTRACT

Objective: A series of 5-substituted-4-amino-1, 2, 4-triazole-linked hydroxamic acid derivatives have been synthesized and explored in vitro to evaluate antibacterial and antifungal activities.

Methods: Different 5-phenyl group substituted-4-amino-1,2,4-triazole-3-thiol reacted with chlorine substituted hydroxamic acid to produce the desired compounds and characterized spectroscopically. Minimum inhibitory concentration (MIC), zone of inhibition (ZOI), growth kinetic studies, and scanning electron microscopy (SEM) were employed to elicit the antimicrobial efficacy of synthesized compounds against a wide range of bacterial and fungal strains.

Results: Compounds 6a, 6b, 6d, and 6k (MIC of $25 \mu \mathrm{g} / \mathrm{ml}$ ) have been found to be more potent against Klebsiella pneumoniae, Bacillus cereus, Bacillus pumilus, Micrococcus luteus, and Pseudomonas aeruginosa, compounds $6 \mathrm{a}-6 \mathrm{~d}, 6 \mathrm{k}$, and $6 \mathrm{l}$ (MIC of 25-50 $\mu \mathrm{g} / \mathrm{ml}$ ) have shown potent antibacterial efficacy against Klebsiella pneumonia, P. aeruginosa, and Vibrio cholera compare to the standard drug amoxicillin (MIC of $60 \mu \mathrm{g} / \mathrm{ml}, 65 \mu \mathrm{g} / \mathrm{ml}$, and $25 \mu \mathrm{g} / \mathrm{ml}$, respectively). Screening for the antifungal activity revealed that the compounds were found to be most active against Candida albicans ( $6 \mathrm{a}$, 6b, and 6l), Candida tropicalis (6b and 6d), and Aspergillus niger (6a, 6b, 6d, and 6j) with MIC of 15-25 $\mu \mathrm{g} / \mathrm{ml}$. Bacteriostatic and fungistatic effect of titled compounds was revealed from growth kinetics study.

Conclusion: Electron donating group at the 5-position of the 5-substituted-1,2,4-triazole-linked hydroxamic acid derivatives conferred the biological effectiveness of the synthesized compounds and also offer a therapeutically effective prototypical structure for further development of new chemical entities with superior antimicrobial activity.

Keywords: 1,2,4-triazole, Hydroxamic acid, Antibacterial, Antifungal.

(C) 2019 The Authors. Published by Innovare Academic Sciences Pvt Ltd. This is an open access article under the CC BY license (http://creativecommons. org/licenses/by/4. 0/) DOI: http://dx.doi.org/10.22159/ajpcr.2019.v12i3.30882

\section{INTRODUCTION}

Themicrobesaregettingresistanttoward theexisting chemotherapeutics in alarming rate which is not only a major concern for public health but also a challenge for the scientific community globally, and the number of cases of multidrug-resistant bacterial infections is increasing nowadays [1]. In clinical practice, the infections caused by the Grampositive bacteria are very common. However, the infection caused by them is sometime severe. Bacillus cereus, Staphylococcus aureus, Bordetella bronchiseptica, Micrococcus luteus, Bacillus pumilus, Bacillus subtilis, and Enterococcus faecalis are some important Gram-positive bacteria causing serious infection in the community, whereas Salmonella typhi, Klebsiella pneumoniae, Vibrio cholerae, Pseudomonas aeruginosa, Escherichia coli, and Salmonella typhimurium are very common Gramnegative bacteria. Among them, E. coli is the most common one. The mortality is higher for infections caused by Gram-negative bacteria than the Gram-positive one. To overcome various microbial infections specifically fungal infection, a large number of triazole drugs have been successfully developed [2]. Azole compounds showed the effect by inhibiting lanosterol $14 \alpha$-demethylase (CYP51) which catalyzes the oxidative removal of the $14 \alpha$-methyl group of lanosterol to give $\Delta^{14,15}$ desaturated intermediates in ergosterol biosynthesis [3] resulting in accumulation of toxic methyl-sterols in membranes leading to fungistatic or fungicidal effect [4]. Some important azoles derivatives such as voriconazole, itraconazole, posaconazole, and fluconazole (Fluc) are used for the treatment of fast-growing fungal infections, which are the leading cause of mortality and morbidity in immunocompromised patients [5]. However, Fluc is not effective against aspergillosis due to its severe drug resistance $[6,7]$.

These scenarios are highlighting the urgent need for novel, efficacious, less toxic, and safe drug candidates in the pipeline. The heterocyclic compounds containing nitrogen, sulfur, and oxygen have an enormous significance in the field of medicinal chemistry [8]. To address the concern, we develop a series of novel 5-substituted-4-amino-1,2,4triazole-linked hydroxamic acid derivatives. Assembling the different pharmacophores in a single frame always leads to compounds having fascinating biological profile [9]. Triazole is one of the pharmacophores in the synthesized compounds. Chemical compounds possess triazole as a core; always play a significant role in the field of medicinal chemistry. Like triazole, imidazole moiety has widely used to synthesize compounds to treat diseases causing by different microorganisms. Even though imidazole and triazole have a similar mode of action, triazoles have several advantages over imidazole, like, oral bioavailability, slow metabolic rate and have minimal effect on sterol synthesis in humans. For these, use of triazole moiety increases rather than the imidazole [10]. Triazole is well known for its antimicrobial property and widely used to treat microorganisms associated diseases [11]. Besides, the antibacterial [12-15] and antifungal [16-19] activity; 1,2,4-triazole possess, anti-inflammatory [20], as well as antioxidants properties [21]. Various well-known drug commercialized in the market possesses 1,2,4-triazole moiety such as Fluc, terconazole, 
itraconazole, [22,23] triazolam [24], and ribavirin [25]. Different $\mathrm{S}$ and N-bridged heterocycles can be synthesized by 1,2,4-triazoles as it possesses nucleophilic centers. Triazolothiazines, triazolothiadiazoles, triazolothiazepines, triazolothiadiazines, and thiazolotriazoles can be synthesized from triazoles, having versatile therapeutic potentials. Another moiety in our synthetically designed compounds is hydroxamic acid. It is another interesting moiety possess antibacterial, antifungal [26,27], anti-inflammatory [28,29], antitumor [30], and anticancer agents [31]. Hydroxamic acid and its derivatives can exert versatile activity by inhibiting several enzymes, such as urease [32], matrix metalloproteinases [33,34], ribonucleotid reductase [35], and 5-lipoxygenase [36]. Hydroxamic acid has the ability to chelate with metals. Few ion exchange resins are built on hydroxamic acids [37]. A number of hydroxamic acid derivatives are used as chemotherapeutic agents such as ibuproxam, hydroxycarbamide, desferrioxamine B, oxametacin, adrafinil, and bufexamac [38]. Hence, considering the above fact we have designed and synthesized some novel 5-substituted-4amino-1,2,4-triazole-linked hydroxamic acid derivatives and evaluate their activity against several Gram-positive, Gram-negative bacteria, and a number of fungal strains.

\section{METHODS}

All the chemicals were procured from Sigma-Aldrich, India, Spectrochem, Merck India Pvt. Ltd., and the media were of purchased from HiMedia. Dichloromethane and dimethyl sulfoxide (DMSO) were procured from Merck, India; all other chemicals used were of analytical grade. In process monitoring of reaction was done on activated silica gel coated plates and the solvent system used was n-hexane:ethylacetate. The melting points of the synthesized compounds were measured by the capillary method and are reported uncorrected. The Fourier-transform infrared (FT-IR) spectra were measured in Nicolet iS10 FT-IR Spectrometer (Thermo Fisher Scientific, USA), ${ }^{1} \mathrm{H}$ nuclear magnetic resonance (NMR) spectra were recorded on AV300 Digital FT NMR Spectrometer, Bruker at 300 MHz using DMSO- $d_{6}$ as the solvent and tetramethylsilane as an internal standard, ${ }^{13} \mathrm{C}$ NMR spectra were obtained at $500 \mathrm{MHz}$ (Bruker, Germany), DMSO- $d_{6}$ as the solvent. Mass spectra $(\mathrm{m} / \mathrm{z})$ of the compounds were recorded on JEOL-JMS 700 spectrometer using electron ionization technique. Scanning electron microscopy (SEM) study of the synthesized compounds was analyzed using SEM JEOL, Tokyo, Japan.

\section{SYNTHETIC PROCEDURE}

General procedure for the synthesis of first intermediate compound (1a-1f)

Substituted-aryl-acid was taken in a $250 \mathrm{ml} \mathrm{rbf.} 40 \mathrm{ml}$ of ethanol was added to it along with $22.5 \mathrm{ml}$ of conc. sulfuric acid. The mixture was refluxed for $2 \mathrm{~h}$ on a steam bath. After that, the mixture was cooled and poured into crushed ice. The mixture was made strongly alkaline by adding sodium carbonate. The mixture was extracted with ether $(3 \times 50 \mathrm{ml})$. The combined ether fraction was collected and kept overnight with a small amount of activated anhydrous sodium sulfate. The ester in the form of oil was collected by means of distilling out the ether.

General procedure for the synthesis of Arylhydrazide (2a-2f)

Esterified compounds (1a-1f) (equimolar) were dissolved in $30 \mathrm{ml}$ of ethanol, and hydrazine hydrate (equimolar) was added dropwise in the mixture with continuous stirring. The resulting mixture was refluxed for $6 \mathrm{~h}$. The solvent was removed by distillation, and the residue was cooled to room temperature. The precipitate formed was filtered and washed subsequently with water, dried, and recrystallized from dehydrated ethanol to yield white crystals.

General procedure for the synthesis of potassium 2-substitutedhydrazine-1-carbodithioate (3a-3f)

Potassium hydroxide (equimolar) was dissolved in $75 \mathrm{ml}$ of absolute alcohol, and arylhydrazide (2a-2f) (equimolar) was added to the above solution. The mixture was then cooled in an ice-bath. To this mixture carbon disulfide was added (equimolar) in small portions with continuous stirring. The reaction mixture was continuously agitated for
$15 \mathrm{~h}$. Finally, the mixture was diluted with $100 \mathrm{ml}$ of anhydrous ether. The residue was filtered, washed with ether $(3 \times 25 \mathrm{ml})$, dried and used as such for the next reaction.

General procedure for synthesis of 4-amino-5-(substitutedphenyl)-4H-1,2,4-triazole-3-thiol (4a-4f)

A suspension of potassium 2-substituted hydrazine-1-carbodithioate (3a-3f) in $20 \mathrm{ml}$ of water and hydrazine hydrate was refluxed for $6 \mathrm{~h}$ with occasional shaking. Next, the hot mixture was cooled to room temperature and diluted with $100 \mathrm{ml}$ of water. Concentrated hydrochloric acid was added dropwise to make the reaction mixture strongly acidic. The precipitate obtained was filtered, washed thoroughly with cold water and dried. The dried product was further recrystallized from dehydrated ethanol to get white crystals.

General procedure for the synthesis of substituted-chloro-Nhydroxyacetamide (5a-5b)

$0.01 \mathrm{~mol}$ of chloroacetylchloride (to synthesize 5a)/3-chloro propionylchloride (to synthesize $5 \mathrm{~b}$ ) was dissolved in $30 \mathrm{ml}$ of methanol. To the reaction mixture, hydroxylamine stock solution ( $0.04 \mathrm{~mol}$ of hydroxylamine hydrochloride) was dissolved in $12 \mathrm{ml}$ of hot methanol. The mixture was stirred for $5 \mathrm{~min}$ and to it, a solution of $0.06 \mathrm{~mol}$ potassium hydroxide in $10 \mathrm{ml}$ of methanol was added dropwise. The resulting solution was cooled at room temperature and filtered to obtain $2 \mathrm{~mol}$ of stock solution was added dropwise and the mixture was stirred for $2 \mathrm{~h}$. The precipitate thus obtained was washed with ether and recrystallized from methanol.

General procedure for the synthesis of title compounds (6a-61)

To an equimolar mixture of 4-amino-5-(substituted-phenyl)-4H-1,2,4triazole-3-thiol and substituted-chloro-N-hydroxypropanamide in acetonitrile, triethylamine was added dropwise and refluxed for 4 h. After cooling down the reaction mixture to the room temperature, $20 \mathrm{ml}$ water was added and extracted with chloroform thrice; aqueous layer was collected and evaporated to produce solid residue, which was further washed with acetone twice and recrystallized from $50 \%$ ethanolic solution to produce white crystals.

\section{Antimicrobial activity}

Microbial strains

In vitro antimicrobial activity was evaluated against 15 different pathogenic bacteria include both Gram-negative such as $S$. typhi 62, K. pneumoniae ATCC 10031, Vibrio cholerae VC 20, P. aeruginosa 25619 , Shigella sonnei NK 4010, E. coli ATCC 25923, and S. typhimurium NTCC 74, and Gram-positive such as Bacillus polymyxa 4747, B. cereus 479, Staphylococcus aureus ATCC 29737, E. faecalis 28, Bordetella bronchiseptica 4617, Micrococcus luteus 10240, B. pumilus 148884, and Bacillus subtilis 6673, respectively, and seven different fungi such as Candida albicans MTCC 183, Candida tropicalis MTCC 2795, Cryptococcus neoformans, Aspergillus niger MTCC 281, Microsporum gypseum, Penicillium chrysogenum, and Cladosporium sp. All these microbial strains were collected from Division of Microbiology and Biotechnology, Department of Pharmaceutical Technology, Jadavpur University, Kolkata, India.

\section{Preparation of inoculums}

Nutrient broth was used to grow the bacterial strains at $37^{\circ} \mathrm{C}$ for $24 \mathrm{~h}$ whereas Czapek Dox media were used to culture the fungal strains at $25-30^{\circ} \mathrm{C}$ for $48-72 \mathrm{~h}$. Afterward, both the bacterial and fungal strains were compared to $0.5 \mathrm{McFarland}$ standards [39] and made the dilution with sterile normal saline was so that produced an initial suspension of $2 \times 10^{6} \mathrm{CFU} / \mathrm{ml}$ for further use.

Determination of minimum inhibitory concentration (MIC)

To determine the MIC of the synthesized compounds agar dilution method was employed as per the National Committee for Clinical Laboratory Standards (NCCLS) 2006 protocol [40]. The synthesized compounds were initially dissolved in a suitable solvent and then serial 
dilution was made to obtain the concentrations $10,25,50,75,100,150$, $200,250,350$, and $400 \mu \mathrm{g} / \mathrm{ml}$. On the separate agar plates with different drug concentrations inocula $\left(2 \times 10^{6} \mathrm{CFU} / \mathrm{mL}\right)$ were spotted and incubated for $24 \mathrm{~h}$ at $37^{\circ} \mathrm{C}$ (for bacterial strains). Amoxicillin (Amx) was used as a reference standard. Whereas, for fungal strains, unlike agar plates, agar media having different drug concentrations were placed as a slant in separate test tubes, and the fungal inocula $\left(2 \times 10^{6} \mathrm{CFU} / \mathrm{mL}\right)$ were streaked and incubated further for $48-72 \mathrm{~h}$ at $28^{\circ} \mathrm{C}$. Fluc was used as a reference standard. MIC was recorded for any lowest concentration where complete growth inhibition of microorganisms was observed visually.

\section{Determination of zone of inhibition (ZOI)}

The well-diffusion method was used to determine the ZOI. For that method, NCCLS 2004 guideline was followed. Briefly, agar plates were prepared, and $100 \mu$ l of bacterial/fungal cell suspension $\left(2 \times 10^{6} \mathrm{CFU} / \mathrm{mL}\right)$ was spread on solid agar plates using a sterile spreader. Sterile borer was used to make the wells, and the tested compounds at their respective MIC was added to the wells and incubated for $24 \mathrm{~h}$ at $37^{\circ} \mathrm{C}$ (for bacteria) and $48-72 \mathrm{~h}$ at $28^{\circ} \mathrm{C}$ (for fungus). Fluc and Amx were used as a reference standard to compare antifungal and antibacterial activity, respectively, of the tested compounds. The zone diameters were measured, and the experiment was performed in triplicates.

\section{Growth kinetic studies}

Growth kinetics studies were carried out to those compounds which have shown higher ZOI at their respective MIC as well as for those have comparable and significant MIC values against the MIC of standard drugs for any bacterial and fungal strains used in the experimental design. Thus, selected tested compounds at their concentration of $2 \times$ MIC were exposed to Gram-positive and Gram-bacteria to carry out the study. For any given bacterial strain, $1 \mathrm{ml}$ overnight cultured bacterial suspension was taken in two separate test tubes containing $4 \mathrm{ml}$ fresh nutrient broth media and incubated for $2-3 \mathrm{~h}$ at $37^{\circ} \mathrm{C}$. After that in one tube, tested compound was added and another tube was used as control (without treatment). At each predetermined time point, $100 \mu \mathrm{l}$ of bacterial suspension was taken from the tubes, diluted in sterile water to achieve the bacterial concentration of $2 \times 10^{6} \mathrm{CFU} / \mathrm{mL}$. From that diluted suspension, $100 \mu \mathrm{l}$ was taken to spread on a solid agar plate and incubated for $24 \mathrm{~h}$ at $37^{\circ} \mathrm{C}$. Afterward, a number of the colony was counted for both test and control [41].

For fungal strains, fungal spores at a concentration of $1 \times 10^{5} \mathrm{CFU} / \mathrm{mL}$ were inoculated $1 \mathrm{~mL}$ of Czapek Dox medium and incubated with or without tested compounds for $48 \mathrm{~h}$ with shaking $(50 \mathrm{rpm})$ at $28^{\circ} \mathrm{C}$. $100 \mu \mathrm{l}$ from each medium was taken to dilute in sterile water and spread onto Czapek Dox agar plate and incubated at $28^{\circ} \mathrm{C}$ for $48-72 \mathrm{~h}$, numbers of the colony-forming unit were counted for test and control [42].

By calculating the viable counts at each time point, a $24 \mathrm{~h}$ time-kill curve was plotted by plotting $\log \mathrm{CFU} / \mathrm{mL}$ against time for bacterial strains and for fungal strains $96 \mathrm{~h}$ time-kill curve were plotted. The experiment was performed in triplicates and expressed as mean \pm SD.

\section{SEM}

Each bacterial and fungal strain was inoculated in two sets, one was treated as test and another was control. After $2 \mathrm{~h}$ tested, compounds were inoculated in tube marked as test. After a sufficient incubation period, cultures were centrifuged at $3000 \mathrm{rpm}$ for $10 \mathrm{~min}$, and the supernatant was discarded to obtain cells pellet. Cells were resuspended in media specific for bacteria and fungus and kept for $10 \mathrm{~min}$ and again centrifuged at $3000 \mathrm{rpm}$ for $10 \mathrm{~min}$. Fixation of cells was done with $4 \%$ glutaraldehyde in $0.1 \mathrm{M}$ phosphate buffer and kept for 2-3 $\mathrm{h}$ and again centrifuged at $3000 \mathrm{rpm}$ for $10 \mathrm{~min}$ and the supernatant was discarded. Cells were washed with sterile water and subjected to dehydration by a series of alcohol washing with increasing strength of $30 \%, 50 \%, 70 \%$, and $100 \%$, respectively. A drop was put on clean glass slide and dried. Platinum coating was done and the sample was examined under SEM [43].

\section{In silico target binding study}

AutoDock Vina [44] using the Lamarckian genetic algorithm for the prediction of binding affinity and searching for the optimum binding site together with the AutoDock Tools (ADT) was employed to set up and perform blind docking calculations of the triazole derivatives binding to substrate binding site of respective enzymes. The membrane protein of S. typhi (protein data bank [PDB] id: 4KR4); chimeric CYP51 (PDB id: chimeric 1EA1); and crystal structure of cytochrome P450 14-alpha-sterol demethylase (Cyp51) from Mycobacterium tuberculosis in complex with azole inhibitors [45] were obtained from the PDB (www.rcsb.org/pdb) [46] at a resolution of $1.60 \AA$ was constructed using AutoDock4 package to study the target binding interaction of all the compounds under study. The coordinates of tested compounds were taken from their two-dimensional structures sketched from ACD ChemSketch Freeware and converted to the PDB format using Open Babel [47].

The target (respective proteins) and the ligand (triazoles) files were prepared using ADT. The water molecules were deleted, and polar hydrogen atoms and Gasteiger charges were added to both the target molecule and the ligands. All other bonds were made rotatable. The target was saved in pdbqt format, and a configuration file was created. Later docking was run. AutoDock generated nine possible binding conformations for each ligand, and the root-mean-square cluster tolerance was set to $2.0 \AA \AA$ in each run. The binding mode and interactions were analyzed for the significant conformers of the compounds under study. All calculations were performed in a Dell system $(3.4 \mathrm{GHz}$ processor, 2GB RAM, 320 GB Hard disk operating system). For each of the docking cases, the lowest energy docked conformation, according to the AutoDock scoring function, was selected as the binding mode. Visualization of the docked pose was done using PyMOL (The PyMOL Molecular Graphics System, Version 1.3, Schrodinger, LLC) molecular graphics program.

Docking was processed with setting of the grid sizes for 4KR4; 30, 26, and 24 and for chimeric 1EA1 40, 34, and 24 along the X-, Y-, and Z-axes with $1 \AA \AA$ spacing which covered all the active residues. In a similar way, the grid center of each target was set for 4KR4; 34.52, 21.86, and 44.96 and for chimeric 1EA1 12.04, 2.87, and $62.50 \AA$ for the protein.

\section{Statistical analysis}

Experimental data were presented as mean standard deviation. One-way analysis of variance was used to determine the statistical significance. $p<0.01$ was considered as statistically significant whereas p $>0.05$ was considered to be statistically not significant.

\section{RESULTS AND DISCUSSION}

The synthetic route of the proposed scaffold was depicted in Scheme 1. A series of novel 2-( (4-amino-5-substituted-4H-1,2,4triazol-3-yl)thio)-N-hydroxyacetamide and 3-((4-amino-5-substituted4H-1,2,4-triazol-3-yl)thio)-N-hydroxypropanamide(6a-6l) compounds were synthesized by reacting 4-amino-5-substituted-4H-1,2,4-triazole3-thiol with the respective 2-chloro-N-hydroxy acetamides/3-chloro- $\mathrm{N}$ hydroxypropanamide (Scheme 1). Briefly, the reaction was carried out in three parts, i.e., synthesis of 4-amino-5-substituted-4H-1,2,4-triazole3-thiol and synthesis of 2-chloro- $\mathrm{N}$-hydroxy acetamides/3-chloro-Nhydroxypropanamide then both were coupled to synthesis of the final compounds. 4-amino-5-substituted-4H-1,2,4-triazole-3-thiol were synthesized by Reid-Heindal method [48], i.e., aryl acids were taken as a starting material and refluxed for $2 \mathrm{~h}$ with ethanol and concentrated sulfuric acid to produce the corresponding aryl esters. The aryl esters were further refluxed with hydrazine hydrate to yield corresponding aryl hydrazides. Then, in the presence of ethanolic potassium hydroxide, the aryl hydrazides were condensed with carbon-di-sulfide to produce potassium-3-aroyldithiocarbazates. These aroyldithiocarbazates were cyclized to produce corresponding aryl substituted 1,2,4-triazole. In another part, chloroacetylchloride or chloropropionylchloride reacted with hydroxyl amine hydrochloride to yield 2-chloro-N-hydroxy 




Scheme 1: Synthetic route for target compounds $6 a-61$

acetamides or 3-chloro-N-hydroxypropanamide, respectively. In the final step, 4-amino-5-(substituted phenyl)-4H-1,2,4-triazole-3-thiol and 2-chloro-N-hydroxy acetamides or 3-chloro-N-hydroxypropanamide were condensed in the presence of triethylamine to produce the final compound, i.e., 2-((4-amino-5-substituted-4H-1,2,4-triazol-3-yl)thio)$\mathrm{N}$-hydroxyacetamide or 3-((4-amino-5-substituted-4H-1,2,4-triazol-3yl)thio)-N-hydroxypropanamide, respectively. All the final compounds (6a-6l) were characterized by FTIR, ${ }^{1} \mathrm{H}$ NMR, ${ }^{13} \mathrm{C}$ NMR, and mass spectral data.

2-((4-amino-5-(pyridin-3-yl)-4H-1,2,4-triazol-3-yl)thio)-Nhydroxyacetamide (6a)

Yield: 1.2 g (70\%), mp: $200-202^{\circ} \mathrm{C}$, FTIR (KBR) $\left(\mathrm{cm}^{-1}\right): 3420.39$ (N-H stretching), 3028.37 (aromatic C-H stretching), 2981.27 (aliphatic C-H stretching), 1431.09 (aromatic $\mathrm{C}=\mathrm{C}$ stretching), 1618.65 (C=0 stretching), 1530.36 ( $\mathrm{C}=\mathrm{N}$ stretching), 1303.91 ( $\mathrm{N}-\mathrm{N}=\mathrm{C}$ stretching). ${ }^{1} \mathrm{H}-\mathrm{NMR}$ (DMSO-d6, $\left.300 \mathrm{MHz}\right) \delta$ ppm: 14.0370 (s; NH), 9.1331 (s; OH), 8.3740-7.5855 (m; Ar-H), $5.7858\left(\mathrm{~s} ; \mathrm{NH}_{2}\right), 1.0373\left(\mathrm{~s} ; \mathrm{CH}_{2}\right) \cdot{ }^{13} \mathrm{C}-\mathrm{NMR}$ (DMSO- $\left.d_{\sigma}\right) \delta$ ppm: $152.4713(\mathrm{CH}), 147.5985(\mathrm{CH}), 124.2174(\mathrm{CH})$, 133.6890 (C), 148.3817 (C), 30.6083 (CH2), 167.2465 (C). Mass: [EI+] $\left(\mathrm{C}_{9} \mathrm{H}_{10} \mathrm{~N}_{6} \mathrm{O}_{2} \mathrm{~S}\right) 266$ Da; calculated for $\mathrm{C}_{9} \mathrm{H}_{10} \mathrm{~N}_{6} \mathrm{O}_{2} \mathrm{~S}, \mathrm{C}, 40.60 ; \mathrm{H}, 3.79 ; \mathrm{N}$, 31.56; O, 12.02; S, 12.04 found: C, 40.72; H, 3.73; N, 31.60; 0, 11.96; S, 11.98 .
3-((4-amino-5-(pyridin-3-yl)-4H-1,2,4-triazol-3-yl)thio)-Nhydroxypropanamide (6b)

Yield: $1 \mathrm{~g}(73 \%), \mathrm{mp}: 197-200^{\circ} \mathrm{C}$, FTIR (KBR) $\left(\mathrm{cm}^{-1}\right): 3412.17(\mathrm{~N}-\mathrm{H}$ stretching), 3026.40 (aromatic C-H stretching), 2930.53 (aliphatic C-H stretching), 1431.34 (aromatic $\mathrm{C}=\mathrm{C}$ stretching), 1618.84 (C=0 stretching), 1530.61 (C=N stretching), 1304.29 ( $\mathrm{N}-\mathrm{N}=\mathrm{C}$ stretching). ${ }^{1} \mathrm{H}-\mathrm{NMR}$ (DMSO-d6, $300 \mathrm{MHz}$ ) $\delta$ ppm: 14.0378 (s; NH), 9.1305 (s; $\mathrm{OH})$, 8.3704-7.5807 (m; Ar-H), $5.7834\left(\mathrm{~s} ; \mathrm{NH}_{2}\right), 1.1560-1.0098(\mathrm{~m}$; $\mathrm{CH}_{2}-\mathrm{CH}_{2}$ ). ${ }^{13} \mathrm{C}$-NMR (DMSO- $d_{6}$ ) $\delta$ ppm: $152.4718(\mathrm{CH}), 147.5995(\mathrm{CH})$, 124.2179 (CH), 133.6898 (C), 148.3825 (C), $30.6088\left(\mathrm{CH}_{2}\right), 25.7294$ $\left(\mathrm{CH}_{2}\right), 167.2482$ (C). Mass: [EI+] $\left(\mathrm{C}_{10} \mathrm{H}_{12} \mathrm{~N}_{6} \mathrm{O}_{2} \mathrm{~S}\right)$ calc. $280.07 \mathrm{Da}$, Found: $280 \mathrm{Da}$; calculated for $\mathrm{C}_{10} \mathrm{H}_{12} \mathrm{~N}_{6} \mathrm{O}_{2} \mathrm{~S}, \mathrm{C}, 42.85 ; \mathrm{H}, 4.32 ; \mathrm{N}, 29.98 ; 0,11.42$; S, 11.44 found: C, 42.92; H, 4.36; N, 29.93; 0, 11.39; S, 11.4 .

2-((4-amino-5-(4-aminophenyl)-4H-1,2,4-triazol-3-yl)thio)-Nhydroxyacetamide (6c)

Yield: $720 \mathrm{mg}$ (768\%), mp: $212-215^{\circ} \mathrm{C}$, FTIR (KBR) $\left(\mathrm{cm}^{-1}\right): 3372.34$ (N-H stretching), 3038.50 (aromatic C-H stretching), 2918.46 (aliphatic C-H stretching), 1499.91 (aromatic $\mathrm{C}=\mathrm{C}$ stretching), 1693.64 ( $\mathrm{C}=\mathrm{O}$ stretching), $1536.51 \quad(\mathrm{C}=\mathrm{N}$ stretching), 1334.46 (N-N=C stretching). ${ }^{1} \mathrm{H}-\mathrm{NMR}$ (DMSO-d6, $\left.300 \mathrm{MHz}\right) \delta$ ppm: 12.80011 (s; $\mathrm{NH}), 9.32056$ (s; OH), 7.62590-6.52428 (m; Ar- H), $5.91882\left(\mathrm{~s} ; \mathrm{NH}_{2}\right.$ ), $1.24555\left(\mathrm{~m} ; \mathrm{CH}_{2}\right) \cdot{ }^{13} \mathrm{C}-\mathrm{NMR}$ (DMSO- $d_{6}$ ) $\delta$ ppm: $116.8490(\mathrm{CH}), 120.9804$ 
$(\mathrm{CH}), 128.4892(\mathrm{CH}), 147.9976(\mathrm{C}), 148.7785(\mathrm{C}), 30.6102\left(\mathrm{CH}_{2}\right)$, 167.2496 (C). Mass: [EI+] $\left(\mathrm{C}_{10} \mathrm{H}_{12} \mathrm{~N}_{6} \mathrm{O}_{2} \mathrm{~S}\right)$ calc. $280.07 \mathrm{Da}$, Found: $280 \mathrm{Da}$; calculated for $\mathrm{C}_{10} \mathrm{H}_{12} \mathrm{~N}_{6} \mathrm{O}_{2} \mathrm{~S}, \mathrm{C}, 42.85 ; \mathrm{H}, 4.32 ; \mathrm{N}, 29.98 ; 0,11.42 ; \mathrm{S}, 11.44$ found: C, 42.53; H, 4.42; N, 29.82; 0, 11.26; S, 11.57 .

3-((4-amino-5-(4-aminophenyl)-4H-1,2,4-triazol-3-yl)thio)-Nhydroxypropanamide (6d)

Yield: $850 \mathrm{mg}$ (71\%), mp: $210-214^{\circ} \mathrm{C}$, FTIR (KBR) $\left(\mathrm{cm}^{-1}\right)$ : 3341.13 (N-H stretching), 3054.69 (aromatic C-H stretching), 2899.90 (aliphatic C-H stretching), 1442.34 (aromatic $\mathrm{C}=\mathrm{C}$ stretching), 1678.97 (C=0 stretching), 1574.30 ( $\mathrm{C}=\mathrm{N}$ stretching), 1271.78 ( $\mathrm{N}-\mathrm{N}=\mathrm{C}$ stretching). ${ }^{1} \mathrm{H}-\mathrm{NMR}$ (DMSO-d6, $\left.300 \mathrm{MHz}\right) \delta$ ppm: 12.7077 (s; NH), 9.4001 (s; OH), 7.7603-7.2099 (m; Ar- H), 5.2626 (s; $\left.\mathrm{NH}_{2}\right), 1.2561\left(\mathrm{~m} ; \mathrm{CH}_{2}-\mathrm{CH}_{2}\right)$. ${ }^{13} \mathrm{C}$-NMR (DMSO-d) $\delta$ ppm: $116.8498(\mathrm{CH}), 128.4889(\mathrm{CH}), 120.9809$ (C), 147.9981 (C), 148.7789 (C), $30.6109\left(\mathrm{CH}_{2}\right), 25.3169\left(\mathrm{CH}_{2}\right)$, 167.2498 (C). Mass: [EI+] $\left(\mathrm{C}_{11} \mathrm{H}_{14} \mathrm{~N}_{6} \mathrm{O}_{2} \mathrm{~S}\right)$ calc. $294.09 \mathrm{Da}$, Found: $294 \mathrm{Da}$; calculated for $\mathrm{C}_{11} \mathrm{H}_{14} \mathrm{~N}_{6} \mathrm{O}_{2} \mathrm{~S}, \mathrm{C}, 44.89 ; \mathrm{H}, 4.79 ; \mathrm{N}, 28.55 ; 0,10.87 ; \mathrm{S}, 10.89$ found: C, 45.05; H, 4.36; N, 28.76; O, 11.09; S, 10.74 .

2-((4-amino-5-(2-mercaptophenyl)-4H-1,2,4-triazol-3-yl)thio)-Nhydroxyacetamide (6e)

Yield: $1.12 \mathrm{~g}(70 \%)$, mp: $105-109^{\circ} \mathrm{C}$, FTIR (KBR) $\left(\mathrm{cm}^{-1}\right)$ : $3367.57(\mathrm{~N}-\mathrm{H}$ stretching), 3082.03 (aromatic C-H stretching), 2980.41 (aliphatic C-H stretching), 1458.46 (aromatic $\mathrm{C}=\mathrm{C}$ stretching), 1696.7 (C=0 stretching), 1563.15 (C=N stretching), 1287.18 ( $\mathrm{N}-\mathrm{N}=\mathrm{C}$ stretching). ${ }^{1} \mathrm{H}-$ NMR (DMSO-d6, 300 MHz) $\delta$ ppm: 13.98857 (s; NH), 9.58518 (s; OH), 8.04105-7.36677 (m; Ar- H), 5.31007 (s; $\mathrm{NH}_{2}$ ), 1.36065 (s; $\mathrm{CH}_{2}$ ). ${ }^{13} \mathrm{C}$-NMR (DMSO- $d$ ) $\delta$ ppm, $125.1776(\mathrm{CH}), 126.1872(\mathrm{CH}), 127.2028$ (CH), 131.2604 (C), 133.5519 (C), 138.5647 (CH), 148.3655 (C), $30.6009\left(\mathrm{CH}_{2}\right), 165.5395$ (C). Mass: [EI+] $\left(\mathrm{C}_{10} \mathrm{H}_{11} \mathrm{~N}_{5} \mathrm{O}_{2} \mathrm{~S}_{2}\right)$ calc. $297.04 \mathrm{Da}$, Found: $297 \mathrm{Da}$; calculated for $\mathrm{C}_{10} \mathrm{H}_{11} \mathrm{~N}_{5} \mathrm{O}_{2} \mathrm{~S}_{2}, \mathrm{C}, 40.39 ; \mathrm{H}, 3.73 ; \mathrm{N}, 23.55$; O, 10.76; S, 21.57 found: C, 40.45; H, 4.34; N, 29.94; O, 11.21; S, 11.52.

3-((4-amino-5-(2-mercaptophenyl)-4H-1,2,4-triazol-3-yl)thio)-Nhydroxypropanamide (6f)

Yield: 1.3 g (74\%), mp: $100-103^{\circ} \mathrm{C}$, FTIR (KBR) $\left(\mathrm{cm}^{-1}\right): 3385.76(\mathrm{~N}-\mathrm{H}$ stretching), 3093.54 (aromatic C-H stretching), 2980.07 (aliphatic C-H stretching), 1458.33 (aromatic $\mathrm{C}=\mathrm{C}$ stretching), 1695.47 (C=O stretching), 1562.97 ( $\mathrm{C}=\mathrm{N}$ stretching), 1286.84 ( $\mathrm{N}-\mathrm{N}=\mathrm{C}$ stretching). ${ }^{1} \mathrm{H}-\mathrm{NMR}$ (DMSO-d6, $300 \mathrm{MHz}$ ) $\delta$ ppm: 14.00011 (s; NH), 9.51002 (s; $\mathrm{OH}$ ), 8.03405-7.33288 (m; Ar- H), 5.30011 (s; $\mathrm{NH}_{2}$ ), 1.37415-1.32802 $\left(\mathrm{m} ; \mathrm{CH}_{2}-\mathrm{CH}_{2}\right.$ ). ${ }^{13} \mathrm{C}$-NMR (DMSO-d $) \delta$ ppm, $125.1786(\mathrm{CH}), 126.1852$ (CH), 127.2035 (CH), 131.2606 (C), 133.5505 (C), $138.5682(\mathrm{CH})$, 148.4652 (C), $30.6089\left(\mathrm{CH}_{2}\right), 25.8079\left(\mathrm{CH}_{2}\right), 165.5396$ (C). Mass: [EI+] $\left(\mathrm{C}_{11} \mathrm{H}_{13} \mathrm{~N}_{5} \mathrm{O}_{2} \mathrm{~S}_{2}\right)$ calc. 311.05Da, Found: $311 \mathrm{Da}$; calculated for $\mathrm{C}_{11} \mathrm{H}_{13} \mathrm{~N}_{5} \mathrm{O}_{2} \mathrm{~S}_{2}, \mathrm{C}, 42.43 ; \mathrm{H}, 4.21 ; \mathrm{N}, 22.49 ; 0,10.28 ; \mathrm{S}, 20.60$ found: $\mathrm{C}$, 41.99; H, 4.28; N, 30.06; 0, 11.39; S, 11.72 .

2-((4-amino-5-(4-nitrophenyl)-4H-1,2,4-triazol-3-yl)thio)-Nhydroxyacetamide $(6 \mathrm{~g})$

Yield: 1.8 g (80\%), mp: $138-140^{\circ} \mathrm{C}$, FTIR (KBR) $\left(\mathrm{cm}^{-1}\right): 3343.96(\mathrm{~N}-\mathrm{H}$ stretching), 3048.17 (aromatic C-H stretching), 2917.92 (aliphatic C-H stretching), 1443.74 (aromatic $\mathrm{C}=\mathrm{C}$ stretching), 1667.23 (C=O stretching), 1514.60 (C=N stretching), 1269.08 (N-N=C stretching). ${ }^{1} \mathrm{H}-\mathrm{NMR}$ (DMSO-d6, $300 \mathrm{MHz}$ ) $\delta$ ppm: 13.64368 (s; NH), 9.30012 (s; $\mathrm{OH}), 7.82233-7.25141$ (m; Ar- H), $5.60085\left(\mathrm{~s} ; \mathrm{NH}_{2}\right), 1.13647\left(\mathrm{~m} ; \mathrm{CH}_{2}\right)$. ${ }^{13} \mathrm{C}$-NMR (DMSO- $\left.d_{6}\right) \delta$ ppm, $129.0487(\mathrm{CH}), 129.2014(\mathrm{CH}), 129.2701$ (CH), 127.9722 (C), 142.9518 (C), 129.3370 (CH), 148.1818 (C), 30.4883 $\left(\mathrm{CH}_{2}\right), 21.0502\left(\mathrm{CH}_{2}\right), 167.2505$ (C). Mass: [EI+] $\left(\mathrm{C}_{11} \mathrm{H}_{13} \mathrm{~N}_{5} \mathrm{O}_{2} \mathrm{~S}\right)$ calc. 279.08 $\mathrm{Da}$, found: $279 \mathrm{Da}$; calculated for $\mathrm{C}_{11} \mathrm{H}_{13} \mathrm{~N}_{5} \mathrm{O}_{2} \mathrm{~S}, \mathrm{C}, 47.30 ; \mathrm{H}, 4.69 ; \mathrm{N}, 25.07 ; \mathrm{O}$, 11.46; S, 11.48 found: C, 48.10; H, 4.18; N, 29.86; 0, 11.14; S, 11.57 .

3 - ((4-amino-5-(p-tolyl)-4H-1,2,4-triazol-3-yl)thio)-Nhydroxypropanamide $(6 \mathrm{~h})$

Yield: $1.4 \mathrm{~g}(73 \%), \mathrm{mp}: 148-150^{\circ} \mathrm{C}$, FTIR (KBR) $\left(\mathrm{cm}^{-1}\right): 3342.98(\mathrm{~N}-\mathrm{H}$ stretching), 3071.72 (aromatic C-H stretching), 2918.91 (aliphatic $\mathrm{C}-\mathrm{H}$ stretching), 1444.07 (aromatic $\mathrm{C}=\mathrm{C}$ stretching), $1667.44 \quad(\mathrm{C}=\mathrm{O}$ stretching), 1515.06 (C=N stretching), 1281.37 ( $\mathrm{N}-\mathrm{N}=\mathrm{C}$ stretching). ${ }^{1} \mathrm{H}-$ NMR (DMSO-d6, $300 \mathrm{MHz}) \delta$ ppm: 13.67881 (s; NH), 9.37850 (s; OH), 7.82335-7.25908 (m; Ar- H), 5.59985 (s; NH ), 1.16979-1.12207 (m; $\mathrm{CH}_{2}-\mathrm{CH}_{2}$ ). ${ }^{13} \mathrm{C}$-NMR (DMSO- $d_{6}$ ) $\delta$ ppm: $129.0512(\mathrm{CH}), 129.2045(\mathrm{CH})$, $129.2723(\mathrm{CH}), 127.9750$ (C), $142.9543(\mathrm{C}), 129.3390(\mathrm{CH}), 148.1827$ (C), $30.4859\left(\mathrm{CH}_{2}\right), 25.8094\left(\mathrm{CH}_{2}\right), 21.0517\left(\mathrm{CH}_{3}\right), 167.2530$ (C). Mass: [EI+] $\left(\mathrm{C}_{12} \mathrm{H}_{15} \mathrm{~N}_{5} \mathrm{O} 2 \mathrm{~S}\right)$ calc. $293.09 \mathrm{Da}$, found: $293 \mathrm{Da}$; calculated for $\mathrm{C}_{12} \mathrm{H}_{15} \mathrm{~N}_{5} \mathrm{O}_{2} \mathrm{~S}, \mathrm{C}, 49.13$; $\mathrm{H}, 5.15$; N, 23.87; 0, 10.91; S, 10.93 found: $\mathrm{C}$, 48.98; H, 5.10; N, 29.95; 0, 10.97; S, 10.99 .

Table 1: MIC values of synthesized compounds (6a-6l)

\begin{tabular}{|c|c|c|c|c|c|c|c|c|c|c|c|c|c|}
\hline \multirow{2}{*}{$\begin{array}{l}\text { Organisms } \\
\text { Bacterial strains }\end{array}$} & \multicolumn{13}{|c|}{$\operatorname{MIC}(\mu \mathrm{g} / \mathrm{ml})$} \\
\hline & $6 a$ & $6 b$ & $6 c$ & 6d & $6 e$ & $6 f$ & $6 \mathrm{~g}$ & $6 h$ & $6 \mathbf{i}$ & $6 \mathbf{j}$ & $6 \mathrm{k}$ & 61 & Amx \\
\hline Salmonella typhi & 25 & 25 & 50 & 50 & - & - & - & - & - & - & 50 & 100 & 10 \\
\hline Klebsiella pneumoniae & 25 & 50 & 25 & 25 & 75 & 100 & - & - & - & - & - & - & 60 \\
\hline Bacillus polymyxa & 100 & 100 & - & - & 50 & 50 & - & - & - & - & 100 & 100 & 32 \\
\hline Bacillus cereus & 50 & 50 & 50 & 25 & - & - & - & - & 50 & 75 & 75 & 50 & 12 \\
\hline Vibrio cholerae & 50 & 75 & - & - & - & - & 75 & 100 & - & - & 50 & 25 & 25 \\
\hline Staphylococcus aureus & 100 & 150 & 100 & 50 & - & - & - & - & - & - & - & - & 16 \\
\hline Enterococcus faecalis & - & - & - & - & - & - & - & - & 200 & 200 & 150 & 100 & 25 \\
\hline Bordetella bronchiseptica & 50 & 50 & - & - & 50 & 50 & - & - & 50 & 100 & - & - & 25 \\
\hline Micrococcus luteus & - & - & - & - & - & - & 100 & 150 & - & - & 25 & 25 & 0.5 \\
\hline Pseudomonas aeruginosa & 25 & 25 & 50 & 50 & - & - & - & - & - & - & 25 & 25 & 65 \\
\hline Shigella sonnei & 100 & 100 & - & - & - & - & - & - & - & - & - & - & 32 \\
\hline Escherichia coli & - & - & 100 & 100 & - & - & 100 & 100 & - & - & 100 & 100 & 10 \\
\hline Bacillus pumilus & 25 & 25 & 50 & 25 & - & - & - & - & 50 & 50 & 50 & 75 & 2 \\
\hline Salmonella typhimurium & - & - & 100 & 200 & 200 & 200 & - & - & - & - & 150 & 100 & 25 \\
\hline Bacillus subtilis & 25 & 25 & 25 & 50 & - & - & 100 & 100 & - & - & 100 & 150 & 8 \\
\hline Fungal stains & $6 a$ & $6 b$ & $6 c$ & 6d & $6 e$ & $6 f$ & $6 \mathrm{~g}$ & $6 h$ & $6 i$ & $6 \mathbf{j}$ & $6 \mathrm{k}$ & 61 & Fluc \\
\hline Candida albicans & 15 & 25 & 50 & 25 & - & - & 100 & 50 & 50 & 100 & 50 & 20 & 12.5 \\
\hline Candida tropicalis & 50 & 20 & 100 & 20 & - & - & 200 & 100 & - & - & - & - & 10 \\
\hline Candida neoformans & 50 & 50 & - & - & - & - & 100 & 200 & 200 & 50 & 50 & 50 & 15 \\
\hline Aspergillus niger & 20 & 20 & 50 & 25 & 300 & 200 & 100 & 50 & 50 & 25 & 50 & 100 & 18 \\
\hline Microsporum gypseum & - & - & 300 & 200 & - & - & 500 & 350 & 500 & 300 & 50 & 50 & 16 \\
\hline Penicillium chrysogenum & - & - & 300 & 250 & - & - & 400 & 400 & - & - & - & - & 56 \\
\hline Cladosporium & 250 & 300 & - & - & - & - & 400 & 200 & 250 & 300 & - & - & 32 \\
\hline
\end{tabular}

Amx: Amoxicillin, Fluc: Fluconazole 


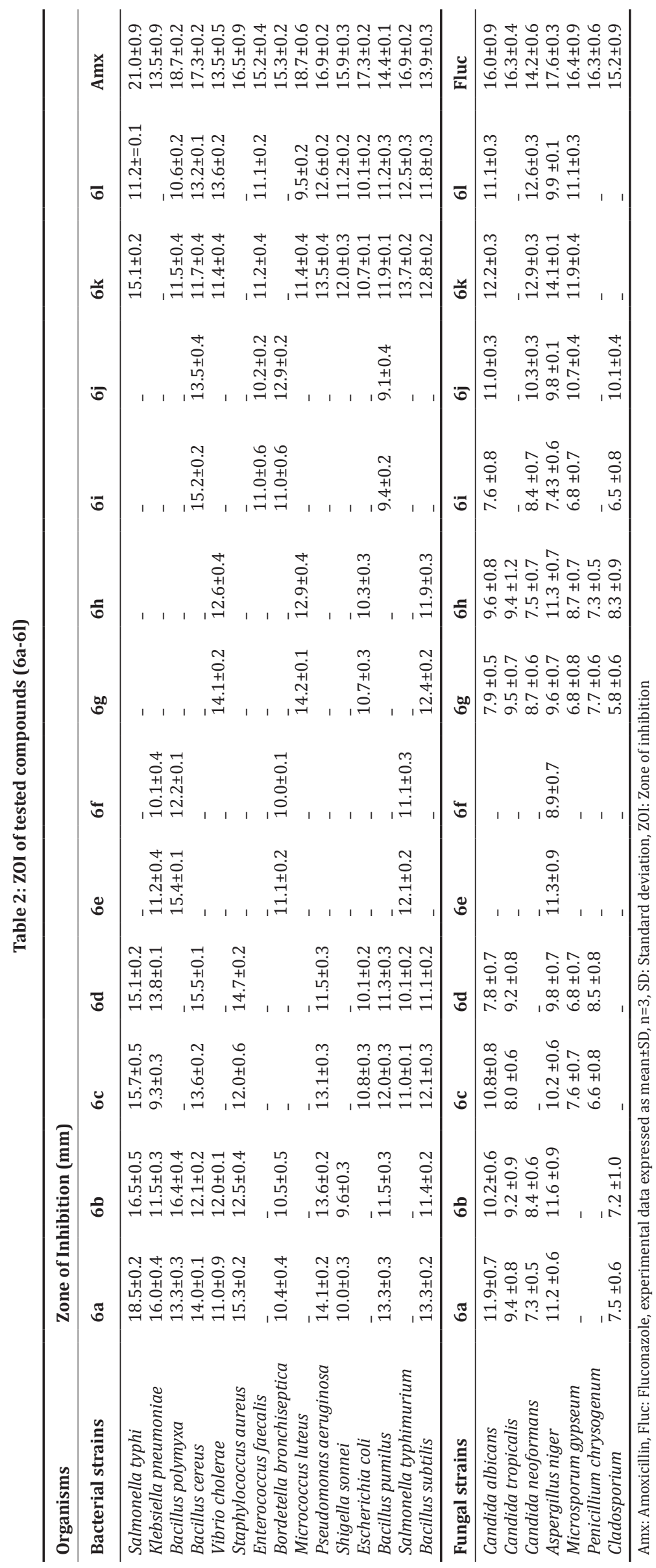


2-((4-amino-5-(4-nitrophenyl)-4H-1,2,4-triazol-3-yl)thio)-Nhydroxyacetamide (6i)

Yield: 1.0 g (67\%), mp: $223-225^{\circ} \mathrm{C}$, FTIR (KBR) $\left(\mathrm{cm}^{-1}\right): 3351.84(\mathrm{~N}-\mathrm{H}$ stretching), 3070.84 (aromatic C-H stretching), 2939.00 (aliphatic C-H stretching), 1451.03 (aromatic $\mathrm{C}=\mathrm{C}$ stretching), $1698.61 \quad(\mathrm{C}=\mathrm{O}$ stretching), 1572.72 (C=N stretching), $1288.70\left(\mathrm{~N}-\mathrm{N}=\mathrm{C}\right.$ stretching). ${ }^{1} \mathrm{H}-$ NMR (DMSO-d6, $300 \mathrm{MHz}$ ) $\delta$ ppm: 14.0948 (s; NH), 9.48836 (s; OH), 8.31648-7.97074 (m; Ar- H), $5.49898\left(\mathrm{~s} ; \mathrm{NH}_{2}\right), 1.19998\left(\mathrm{~m} ; \mathrm{CH}_{2}\right)$. ${ }^{13} \mathrm{C}$-NMR (DMSO- $d_{6}$ ) $\delta$ ppm: $122.0874(\mathrm{CH}), 128.4884(\mathrm{CH}), 136.8090$ (C), 147.9985 (C), 148.7815 (C), $30.6092\left(\mathrm{CH}_{2}\right), 167.2478$ (C). Mass: [EI+] $\left(\mathrm{C}_{10} \mathrm{H}_{10} \mathrm{~N}_{6} \mathrm{O}_{4} \mathrm{~S}\right)$ calc. $310.05 \mathrm{Da}$, Found: $310 \mathrm{Da}$; calculated for $\mathrm{C}_{10} \mathrm{H}_{10} \mathrm{~N}_{6} \mathrm{O}_{4} \mathrm{~S}, \mathrm{C}, 38.71 ; \mathrm{H}, 3.25 ; \mathrm{N}, 27.08 ; 0,20.63 ; \mathrm{S}, 10.33$ found: $\mathrm{C}$, 39.76; H, 2.95; N, 26.73; O, 20.48; S, 10.13

3-((4-amino-5-(4-nitrophenyl)-4H-1,2,4-triazol-3-yl)thio)-Nhydroxypropanamide (6j)

Yield: 1.2 g (71\%), mp: $212-215^{\circ} \mathrm{C}$, FTIR (KBR) $\left(\mathrm{cm}^{-1}\right): 3365.73(\mathrm{~N}-\mathrm{H}$ stretching), 3076.11 (aromatic $\mathrm{C}-\mathrm{H}$ stretching), 2857.26 (aliphatic C-H stretching), 1475.39 (aromatic $\mathrm{C}=\mathrm{C}$ stretching), 1681.59 (C=0 stretching), 1519.24 ( $\mathrm{C}=\mathrm{N}$ stretching), 1311.25 ( $\mathrm{N}-\mathrm{N}=\mathrm{C}$ stretching). ${ }^{1} \mathrm{H}-\mathrm{NMR}$ (DMSO-d6, $300 \mathrm{MHz}$ ) $\delta$ ppm: 14.1975 (s; NH), 9.15008 (s; OH), 8.32100-7.98191 (m; Ar- H), 5.48758 (s; $\mathrm{NH}_{2}$ ), 1.20831-1.16040 (m; $\mathrm{CH}_{2}-\mathrm{CH}_{2}$ ). ${ }^{13} \mathrm{C}$-NMR (DMSO-d $d_{6}$ ) $\delta$ ppm: $122.0878(\mathrm{CH}), 128.4887(\mathrm{CH})$, 136.8098 (C), 147.9989 (C), 148.7818 (C), $30.6095\left(\mathrm{CH}_{2}\right), 25.8370$ $\left(\mathrm{CH}_{2}\right), 167.2482$ (C). Mass: [EI+] $\left(\mathrm{C}_{11} \mathrm{H}_{12} \mathrm{~N}_{6} \mathrm{O}_{4} \mathrm{~S}\right)$ calc. 324.06 Da, found: $324 \mathrm{Da}$; calculated for $\mathrm{C}_{11} \mathrm{H}_{12} \mathrm{~N}_{6} \mathrm{O}_{4} \mathrm{~S}, \mathrm{C}, 40.74 ; \mathrm{H}, 3.73 ; \mathrm{N}, 25.91 ; \mathrm{O}, 19.73$; S, 9.89 found: C, 41.14; H, 3.58; N, 26.06; O, 19.95; S, 10.07 .

2-((4-amino-5-(3-nitrophenyl)-4H-1,2,4-triazol-3-yl)thio)-Nhydroxyacetamide (6k)

Yield: $1 \mathrm{~g}(70 \%), \mathrm{mp}: 220-224^{\circ} \mathrm{C}$, FTIR (KBR) $\left(\mathrm{cm}^{-1}\right): 3308.80(\mathrm{~N}-\mathrm{H}$ stretching), 3082.67 (aromatic C-H stretching), 2926.24 (aliphatic C-H stretching), 1481.03 (aromatic $\mathrm{C}=\mathrm{C}$ stretching), $1700.90 \quad(\mathrm{C}=0$ stretching), 1518.48 (C=N stretching), 1296.15 ( $\mathrm{N}-\mathrm{N}=\mathrm{C}$ stretching). ${ }^{1} \mathrm{H}-\mathrm{NMR}$ (DMSO-d6, $300 \mathrm{MHz}$ ) $\delta$ ppm: 14.1101 (s; NH), 9.4886 (s; OH), 8.9514-7.8044 (m; Ar- H), 5.8517 (s; $\left.\mathrm{NH}_{2}\right), 1.2327$ (s; $\left.\mathrm{CH}_{2}\right) .{ }^{13} \mathrm{C}-\mathrm{NMR}$ (DMSO- $d_{6}$ ) $\delta$ ppm: $121.0683(\mathrm{CH}), 131.2639(\mathrm{CH}), 132.0087$ (C), $133.0016(\mathrm{CH}), 148.0011(\mathrm{C}), 148.9088(\mathrm{C}), 31.8313\left(\mathrm{CH}_{2}\right), 165.7338$ (C). Mass: [EI+] $\left(\mathrm{C}_{10} \mathrm{H}_{10} \mathrm{~N}_{6} \mathrm{O}_{4} \mathrm{~S}\right)$ calc. $310.05 \mathrm{Da}$, found: $310 \mathrm{Da}$; calculated for $\mathrm{C}_{10} \mathrm{H}_{10} \mathrm{~N}_{6} \mathrm{O}_{4} \mathrm{~S}, \mathrm{C}, 38.71 ; \mathrm{H}, 3.25 ; \mathrm{N}, 27.08 ; \mathrm{O}, 20.63 ; \mathrm{S}, 10.33$ found: $\mathrm{C}$, 38.80; H, 3.29; N, 26.98; 0, 20.61; S, 10.30 .

3-((4-amino-5-(3-nitrophenyl)-4H-1,2,4-triazol-3-yl)thio)-Nhydroxypropanamide (61)

Yield: 1.1 g (70\%), mp: $210-212^{\circ} \mathrm{C}$, FTIR (KBR) $\left(\mathrm{cm}^{-1}\right): .{ }^{1} \mathrm{H}-\mathrm{NMR}$ (DMSO-d6, 300 MHz) $\delta$ ppm: 14.1199 (s; NH), 9.4883 (s; OH), 8.94887.1773 (m; Ar- H), 5.8455 (s; $\mathrm{NH}_{2}$ ), 2.2546 (s; $\mathrm{CH}_{2}$ ), 1.2186 (s; $\mathrm{CH}_{2}$ ). ${ }^{13} \mathrm{C}$-NMR (DMSO- $d_{6}$ ) $\delta$ ppm: $121.0678(\mathrm{CH}), 131.2635(\mathrm{CH}), 132.0088$ (C), $133.0010(\mathrm{CH}), 148.0003$ (C), 148.9085 (C), $31.8309\left(\mathrm{CH}_{2}\right), 25.9689$ (CH2), 165.7335 (C). Mass: [EI+] $\left(\mathrm{C}_{11} \mathrm{H}_{12} \mathrm{~N}_{6} \mathrm{O}_{4} \mathrm{~S}\right)$ calc. $324.06 \mathrm{Da}$, found: $324 \mathrm{Da}$; calculated for $\mathrm{C}_{11} \mathrm{H}_{12} \mathrm{~N}_{6} \mathrm{O}_{4} \mathrm{~S}, \mathrm{C}, 40.74 ; \mathrm{H}, 3.73 ; \mathrm{N}, 25.91 ; \mathrm{O}, 19.73$; S, 9.89 found: C, 40.64; H, 3.67; N, 25.99; 0, 19.79; S, 9.92.

The synthesized compounds (6a-6l) were exposed to 15 different bacterial strains including both Gram-negative and Gram-positive bacteria and found to be efficacious at a range of MIC values between 25 and $200 \mu \mathrm{g} / \mathrm{ml}$. Amx was used as a reference standard to compare the antibacterial activity of the synthesized compounds. The comparative MIC values of the compounds were tabulated in Table 1 . The results showed that majority of the synthesized compounds elicited variable degrees of inhibition against the tested microorganisms. Compounds 6a, 6b, 6c, 6d, 6k, and $6 \mathrm{l}$ exhibited potent antibacterial activity against K. pneumonia, B. cereus, P. aeruginosa, B. pumilus, S. typhi, B. subtilis, and V. cholerae at a MIC of $25 \mu \mathrm{g} / \mathrm{ml}$ whereas compound $6 \mathrm{e}(75 \mu \mathrm{g} / \mathrm{ml})$ has shown moderate activity compared to the standard having the MIC of $60 \mu \mathrm{g} / \mathrm{ml}$. Compound $6 \mathrm{f}(100 \mu \mathrm{g} / \mathrm{ml})$ has poor efficacy against the same strain where other compounds have shown no activity. Against P. aeruginosa, compounds $6 \mathrm{a}-6 \mathrm{~d}, 6 \mathrm{k}$, and $6 \mathrm{l}$ (MIC of $25-50 \mu \mathrm{g} / \mathrm{ml}$ ) have found to be more potent than standard compound $(65 \mu \mathrm{g} / \mathrm{ml})$ where other compounds have found to be no activity. Against the other bacterial strain compounds have shown moderate to less activity compared to the standard like, compound $6 \mathrm{e}, 6 \mathrm{f}(50 \mu \mathrm{g} / \mathrm{ml})$ against B. polymyxa; compounds $6 \mathrm{a}, 6 \mathrm{k}$, and $6 \mathrm{l}(50 \mu \mathrm{g} / \mathrm{ml})$ against $V$. cholerae; compounds $6 \mathrm{a}, 6 \mathrm{~b}, 6 \mathrm{e}, 6 \mathrm{f}$, and $6 \mathrm{i}(50 \mu \mathrm{g} / \mathrm{ml})$ against $B$. bronchiseptica were moderately active where other compounds have mild bioactivity against the tested bacterial strains, however, compared to the standard they have very less to no activity.

In vitro, antifungal activity was evaluated against seven different fungal strains and the result showed that the synthesized compounds were active against all tested fungi to some extent (Table 1). Among the tested compounds, compound 6 a showed potent activity against Candida albicans at MIC $15 \mu \mathrm{g} / \mathrm{ml}$ which is nearer to standard Fluc $(12.5 \mu \mathrm{g} / \mathrm{ml})$, whereas compound $6 \mathrm{l}(20 \mu \mathrm{g} / \mathrm{ml})$ was found to be moderately active against Candida albicans. Against A. niger compounds $6 \mathrm{a}$ and $6 \mathrm{~b}(20$ $\mu \mathrm{g} / \mathrm{ml})$ were potent whereas compounds $6 \mathrm{~d}$ and $6 \mathrm{j}(25 \mu \mathrm{g} / \mathrm{ml})$ were moderately active compared to the standard $(18 \mu \mathrm{g} / \mathrm{ml})$. On the other hand, compounds $6 \mathrm{~b}$ and $6 \mathrm{~d}(20 \mu \mathrm{g} / \mathrm{ml})$ elicited moderate activity against Candida tropicalis.

The ZOI of the compounds which showed inhibitory efficacy against individual strains of bacteria and fungus, was calculated at their respective MIC and tabulated in Table 2 like, against $S$. typhi compounds $6 \mathrm{a}$ and $6 \mathrm{~b}$ showed the ZOI of $18.5 \pm 0.2 \mathrm{~mm}$ (at MIC of $25 \mu \mathrm{g} / \mathrm{ml}$ ) and $16.5 \pm 0.5 \mathrm{~mm}$ (at MIC of $25 \mu \mathrm{g} / \mathrm{ml}$ ), respectively. Against K. pneumoniae compounds $6 \mathrm{a}, 6 \mathrm{c}$, and $6 \mathrm{~d}$ showed ZOI of $16.0 \pm 0.4 \mathrm{~mm}, 9.3 \pm 0.3 \mathrm{~mm}$, and $13.8 \pm 0.1 \mathrm{~mm}$ (at MIC of $25 \mu \mathrm{g} / \mathrm{ml}$ ), respectively. Compound 6e showed ZOI of $15.4 \pm 0.1 \mathrm{~mm}$ (MIC $=50 \mu \mathrm{g} / \mathrm{ml}$ ) against $B$. polymyxa. Compound 61 have a ZOI of $13.6 \pm 0.2 \mathrm{~mm}(\mathrm{MIC}=25 \mu \mathrm{g} / \mathrm{ml}$ ) against $V$. cholerae. ZOI of $14.7 \pm 0.2 \mathrm{~mm}$ (MIC $=50 \mu \mathrm{g} / \mathrm{ml}$ ) for compound $6 \mathrm{~d}$ against $S$. aureus. $11.4 \pm 0.4 \mathrm{~mm}$ and $9.5 \pm 0.2 \mathrm{~mm}$ (MIC $=25 \mu \mathrm{g} / \mathrm{ml}$ ) for compounds $6 \mathrm{k}$ and $6 \mathrm{l}$ against M. luteus. For compounds $6 \mathrm{a}, 6 \mathrm{~b}, 6 \mathrm{k}$, and $6 \mathrm{l}$, ZOI was almost $13 \mathrm{~mm}$ against $P$. aeruginosa at MIC of $25 \mu \mathrm{g} / \mathrm{ml}$. ZOI of $13.3 \pm 0.3 \mathrm{~mm}$ and $13.3 \pm 0.2 \mathrm{~mm}$ were higher for compound $6 \mathrm{a}$ against $B$. pumilus and $B$. subtilis, respectively, at MIC of $25 \mu \mathrm{g} / \mathrm{ml}$.

For fungal strains, it was observed that compound $6 \mathrm{a}$ has the ZOI of $11.9 \pm 0.7 \mathrm{~mm}$ (MIC $=15 \mu \mathrm{g} / \mathrm{ml}$ ) for C. albicans; compound $6 \mathrm{~b}$ showed $9.2 \pm 0.9 \mathrm{~mm}(\mathrm{MIC}=20 \mu \mathrm{g} / \mathrm{ml}$ ) for $C$. tropicalis, and against A. niger compounds $6 \mathrm{a}$ and $6 \mathrm{~b}$ have shown almost similar ZOI of $11.2 \pm 0.6 \mathrm{~mm}$ and $11.6 \pm 0.9 \mathrm{~mm}$, respectively, at the MIC of $20 \mu \mathrm{g} / \mathrm{ml}$.

Compounds $6 \mathrm{a}, 6 \mathrm{~b}$, and $6 \mathrm{l}$ were most active against the fungal strain C. albicans. It was found very interesting that all of the synthesized compounds were active against $A$. niger even compounds $6 \mathrm{e}$ and $6 \mathrm{f}$ which were not effective against the any of the fungal strain (between the tested concentration to determine MIC) used for this experimental design, were active against $A$. niger although the MIC values were high enough, i.e., $300 \mu \mathrm{g} / \mathrm{ml}$ and $200 \mu \mathrm{g} / \mathrm{ml}$, respectively, compared to the standard drug Fluc $(18 \mu \mathrm{g} / \mathrm{ml})$. However, compounds $6 \mathrm{~b}$ and $6 \mathrm{a}$ showed potent antifungal activity against $A$. niger with ZOI of $11.6 \pm 0.9 \mathrm{~mm}$ and $11.2 \pm 0.6 \mathrm{~mm}$ at the MIC of $20 \mu \mathrm{g} / \mathrm{ml}$.

SEM was carried out to assess morphological changes in bacterial cells following the exposure of most active compounds against selective bacterial as well as fungal strains; like, for bacterial strains, B. pumilus was treated with compound 6 a and P. aeruginosa was treated with compound $6 \mathrm{~b}$ whereas for fungal strains, $C$. tropicalis was treated with compound $6 \mathrm{~b}$ and $C$. albicans was treated with compound $6 \mathrm{a}$. The SEM analysis showed clear morphological changes in bacterial cells when treated with tested compounds. Fig. $1 \mathrm{k}$ and 1 depict the SEM image of control and treated B. pumilus, respectively. SEM micrographs of B. pumilus revealed that when the bacterium was exposed to the tested compound, the cells disrupted following alterations in the cell integrity. $P$. aeruginosa when treated with the tested compound (Fig. 1n), appeared to swell with loss of its normal morphology as depicted by the control (Fig. $1 \mathrm{~m}$ ). Moreover, based on the pathogenic mechanisms of B. pumilus and P. aeruginosa, an alternative strategy for the treatment 


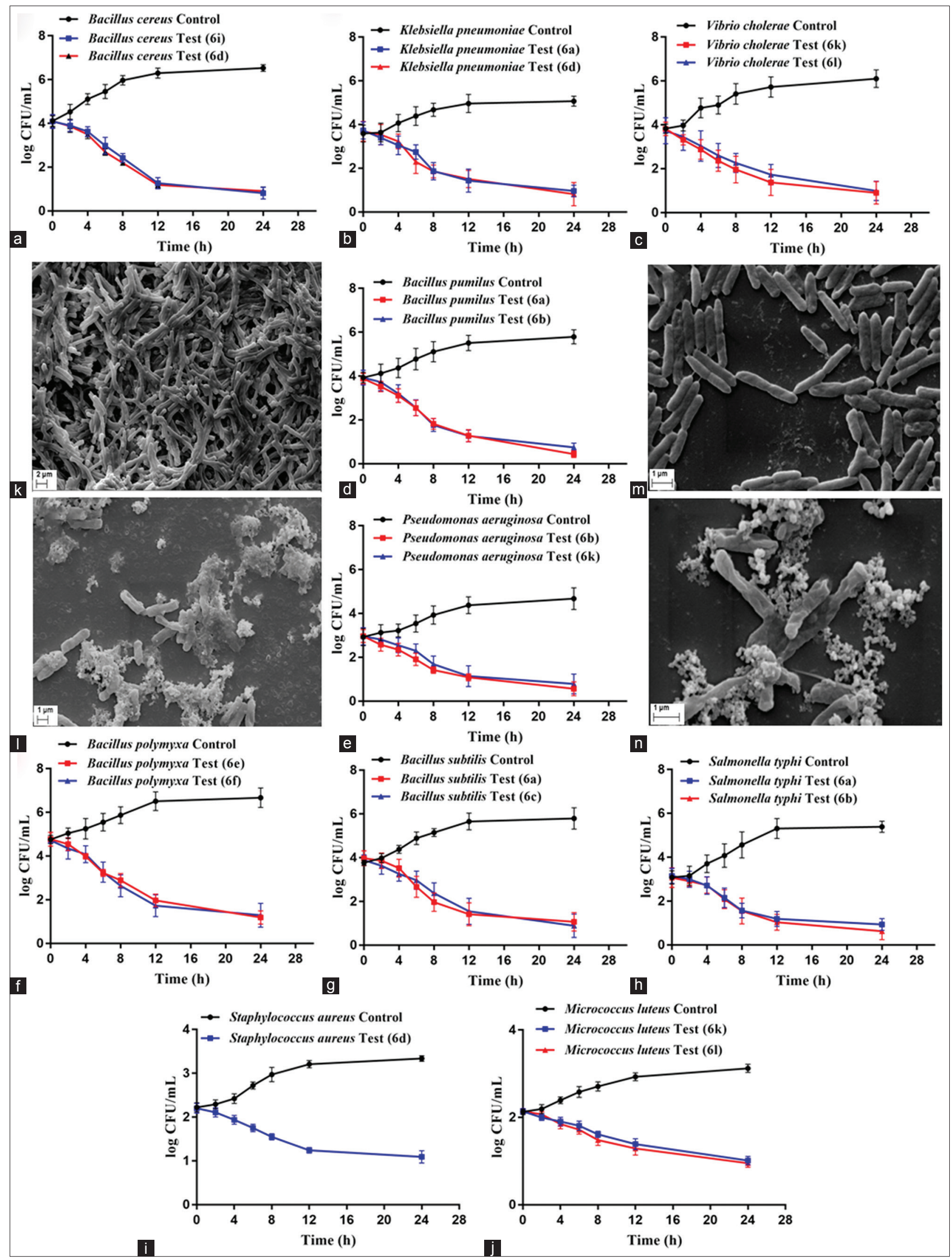

Fig. 1: Time-dependent in vitro growth curve of Bacillus cereus (a), Klebsiella pneumoniae (b), Vibrio cholerae (c), Bacillus pumilus (d), Pseudomonas aeruginosa (e), Bacillus polymyxa (f), Bacillus subtilis (g), Salmonella typhi (h), Staphylococcus aureus (i), Micrococcus luteus ( $\mathrm{j}$ ) at their $2 \times$ MIC values against test compounds and scanning electron micrograph of $B$. pumilus (k) before treatment, (l) after treatment; $P$. aeruginosa $(\mathrm{m})$ before treatment, (n) after treatment 

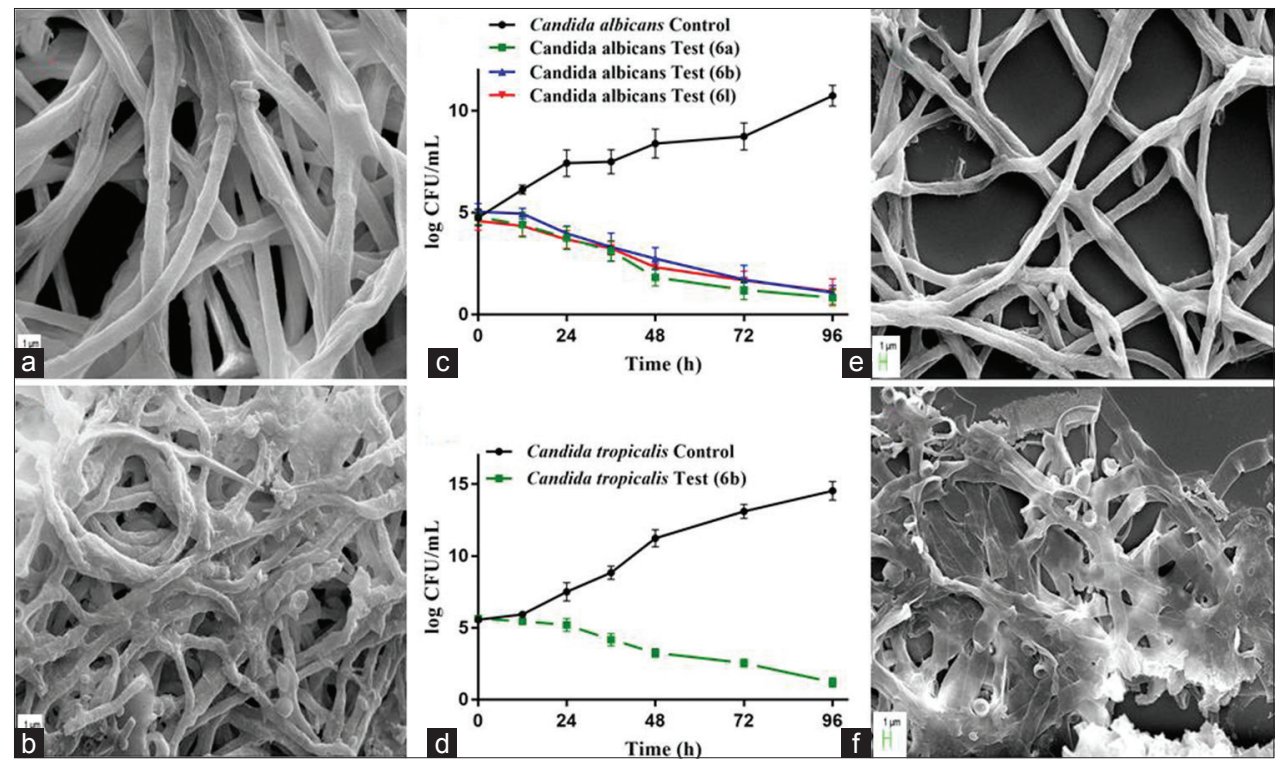

Fig. 2: Scanning electron micrograph of Candida tropicalis (a) before treatment, (b) after treatment; Candida albicans (e) before treatment, (f) after treatment and time-dependent in vitro growth curve of $C$. albicans (c) and $C$. tropicalis (d) at their $\times 2$ MIC values against test compounds

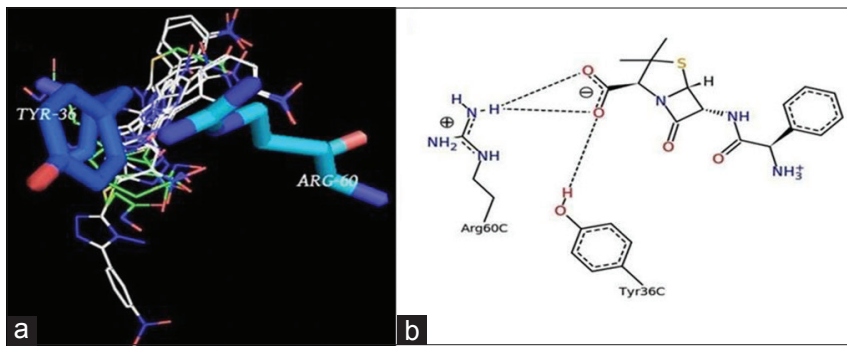

Fig. 3: (a) Cluster of conformers within the active site of the target protein of Salmonella typhi (pdb id: 4kr4), (b) PoseView of standard ampicillin within the active site of $4 \mathrm{kr} 4$

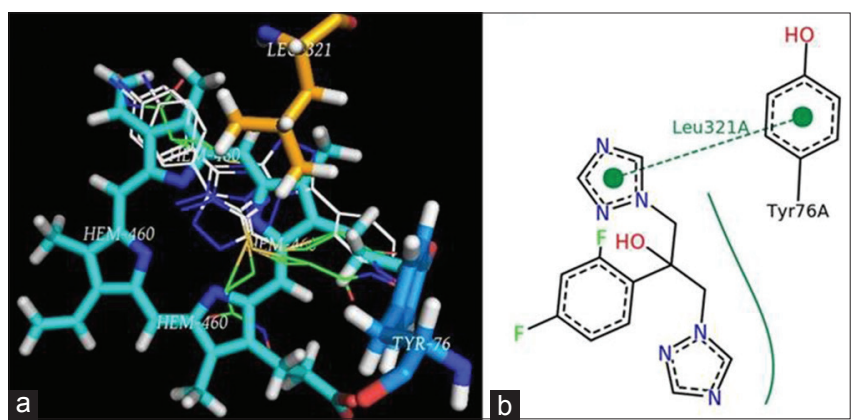

Fig. 4: (a) Cluster of conformers within the active site of the target enzyme of Candida albicans (pdb id: Chimeric 1ea1), (b) PoseView of standard fluconazole within the active site of $4 \mathrm{kr} 4$

of the infections caused by these organisms is of supreme importance in the current scenario.

Fig. 2a and b are the SEM images of control and treated Candida tropicalis, respectively, whereas, Fig. 2e and f show the SEM images of control and treated Candida tropicalis, respectively. In both the cases, structural changes in the SEM studies appeared progressively due to cohesion, diffusibility, agglomeration, and ultimate structure distortion and disintegration.

Growth kinetic study was done against the bacterial strains such as B. cereus, K. pneumoniae, V. cholerae, B. pumilus, P. aeruginosa,
B. polymyxa, B. subtilis, S. typhi, S. aureus, and M. luteus for the tested compounds which showed higher ZOI at their respective MIC (Fig. 1a$\mathrm{J}$, respectively). Whereas, for fugal strains, compounds $6 \mathrm{a}, 6 \mathrm{~b}$, and $6 \mathrm{l}$ were studied for the growth kinetics evaluation against $C$. albicans and compound $6 \mathrm{~b}$ for $C$. tropicalis displayed in Fig. 2c and d, respectively. Although almost all synthesized compounds have shown antifungal activity against $A$. niger, the growth kinetic study could not be performed as the A. niger strains forming very dark brown spores which could not be counted visually. From the growth kinetics data of bacteria as well as fungus from Figs. 1 and 2, respectively, it could be observed that the all best active compounds showed bacteriostatic and fungistatic activity.

It could be observed from the antimicrobial data that synthesized aryl substituted 1,2,4- triazole-linked hydroxamic acid derivatives showed moderate to high activity against tested bacterial as well as fungal strains. The antifungal enzyme, lanosterol-14 $\alpha$-demethylase is one of the primary targets of antifungal drug discovery. The molecular mechanism of triazole as an antifungal has already been established. All the compounds considered for the study possessed 1,2,4-triazole ring in it; therefore, all the compounds exhibited affinity toward the enzyme. The intensity of binding was high in compounds $6 \mathrm{a}, 6 \mathrm{~b}$, and $6 \mathrm{l}$, which may be due to the ring nitrogen and the electronegative nitro group present in the aryl group attached to the central triazole, respectively. Triazole is well known for its antimicrobial activity. However, electronrich aryl substitutions at 5- position of the 1,2,4-triazole could be an added benefit along with the linked hydroxamic acid. For many decades, the antimicrobial activity of different hydroxamic acid derivatives was evaluated. It was reported that hydroxamic acid is potent as well as a specific inhibitor of bacterial urease [49]. Hence, the antimicrobial activity of the synthesized compounds might be due to the partial effect of hindered urea metabolism by the hydroxamic acid part. It was also reported that the presence of - $\mathrm{CONHOH}$ group is very much necessary for the inhibition of microbial urease. In addition, alkyl group present in the hydroxamic acid part of the synthesized compounds influenced the effectiveness.

The binding mode of the highest active test compound exhibiting appreciable antimicrobial activity was investigated by docking studies. Crystal structure of Salmonella typhi was obtained from protein repository. On the other hand, no experimental structural information on the active site of the target enzyme Candida P450DM is available. It is observed from an extensive literature search that high homology exists 
between the mycobacterium P450DM and Candida P450DM. Following the method of Rossello et al. [50], the chimeric enzyme for the Candida albicans (CACYP51) was developed from that of mycobacterium P450DM (MT CYP51) extracted from the PDB (entry code 1EA1). The residues that were arranged in a range of $7 \AA$ from Fluc were substituted with those of Candida P450DM. Substitutions were made by replacement of the residues Pro77, Phe78, Met79, Arg96, Met99, Leu100, Phe255, Ala256, His258, Ile322, Ile323, and Leu324 by Lys77, His78, Leu79, Leu96, Lys99, Phe100, Met255, Gly256, Gln258, His322, Ser323, and Ile324, which were thought to be necessary for the ligandreceptor interaction. Compound 6k, 2-((4-amino-5-(3-nitrophenyl)$4 \mathrm{H}-1,2,4$-triazol-3-yl)thio)-N-hydroxyacetamide was within the active site of $4 \mathrm{kr} 4$. The active site composition was procured from rcsb and the binding pattern of ampicillin in Fig. 3b was set as standard. Almost all the docked conformers occupied the proximal vicinity of the active site which is clearly understood from Fig. 3a.

Based on the fact that azoles exhibit antifungal activity by inhibition of a cytochrome P450 enzyme, lanosterol-14- $\alpha$-demethylase during the biosynthesis of ergosterol leading to accumulation of 14- $\alpha$-sterols in the fungal cell membrane causing destabilization and membrane degradation, it was thought worthwhile to investigate the binding mode of the highestactive test compound, 6c, 2-((4-amino-5-(4-aminophenyl)$4 \mathrm{H}-1,2,4$-triazol-3-yl)thio)-N-hydroxyacetamide in the active site of chimeric enzyme of cytochrome P450-14- $\alpha$-sterol demethylase from Candida albicans (Candida P450DM) (chimeric 1EA1).

Binding mode analysis of test compound $6 \mathrm{c}$ in the active site of chimeric 1EA1 (Fig. 4a) showed that the test compound was very close to the porphyrin ring, HEM 470, similar as that of the cocrystallized ligand Fluc. Docking poses of the test compound suggest the presence of active site residues leu321, tyr76 as in case of Fluc (Fig. 4b).

\section{CONCLUSION}

A series of twelve 5-substituted-1,2,4-triazole-linked hydroxamic acid derivatives (6a-6l) were synthesized and evaluated for their in vitro antimicrobial potency and found to be potent to moderately active in inhibiting the pathogenic growth. The significant antimicrobial property may be attributed to the putative substructure of triazole (which itself can form ionic interaction with the various microbial target; the most acceptable is lanosterol-14 $\alpha$-demethylase in Candida albicans) and the salient feature of the hydroxamic group. The aryl group with its substituent leaves a mixed opinion with respect to the biological interaction. The electron donating ability of methyl, mercapto, amino group strengthen the binding affinity of the aryl group with the active site of the target might be one of the strong supporting evidence for their potency, but in the same ground how this nitro group being a strong electron group helps in lifting the chemotherapeutic index is difficult to establish. Therefore, the series requires to be well extended. Thus, it could be concluded that the synthesized 1,2,4-triazole-linked hydroxamic acid derivatives with electron donating groups at 5-position of the 1,2,4-triazole moiety may provide a therapeutically effective chemical framework from which potential antimicrobial agents may be developed further. Therefore, further optimizations of this prototypical molecular framework with some diversified molecular fragments may generate new drug entities having potent antimicrobial activity.

\section{ACKNOWLEDGMENTS}

This experimental work was supported by the Indian Council of Medical Research (ICMR), India. Mousumi Das is a senior research fellow of ICMR (No. 45/9/13-PHA/BMS). The authors are grateful to UPE Phase - II, University Grants Commission, Government of India, for providing instrumental support.

\section{AUTHORS' CONTRIBUTIONS}

All authors have made considerable contributions to the work reported in the manuscript.

\section{CONFLICTS OF INTEREST}

The authors have no conflicts of interest.

\section{REFERENCES}

1. Pisal P, Deodhar M, Kale A, Nigade G, Pawar S. Design, synthesis, docking studies and biological evaluation of 2-phenyl-3-(substituted benzo[d] thiazol-2-ylamino)-quinazoline-4(3h)-one derivativesas antimicrobial agents. Int J Pharm Pharm Sci 2018;10:57-61.

2. Gadhave PP, Dighe NS, Pattan SR, Deotarse P, Musmade DS, Shete RV. Current biological and synthetic profile of triazoles: A review. Ann Biol Res 2010;1:82-9.

3. Aoyama Y, Yoshida Y, Sato R. Yeast cytochrome P-450 catalyzing lanosterol 14 alpha-demethylation. II. Lanosterol metabolism by purified P-450(14)DM and by intact microsomes. J Biol Chem 1984;259:1661-6.

4. Kelly SL, Arnoldi A, Kelly DE. Molecular genetic analysis of azole antifungal mode of action. Biochem Soc Trans 1993;21:1034-8.

5. Groll AH, Lumb J. New developments in invasive fungal disease. Future Microbiol 2012;7:179-84.

6. Casalinuovo IA, Di Francesco P, Garaci E. Fluconazole resistance in Candida albicans: A review of mechanisms. Eur Rev Med Pharmacol Sci 2004;8:69-77.

7. Hoffman HL, Ernst EJ, Klepser ME. Novel triazole antifungal agents. Expert Opin Investig Drugs 2000;9:593-605.

8. Bairam R, Srinivasa MM, Sreekanth S. Synthesis, characterization, biological evaluation and docking of some novel substituted 1, 3-thiazine derivatives. Int J Pharm Pharm Sci 2017;9:233-42.

9. Aggarwal N, Kumar R, Srivastva C, Dureja P, Khurana JM. Synthesis of nalidixic acid based hydrazones as novel pesticides. J Agric Food Chem 2010;58:3056-61.

10. Palekar VS, Damle AJ, Shukla SR. Synthesis and antibacterial activity of some novel bis-1,2,4-triazolo[3,4-b]-1,3,4-thiadiazoles and bis-4thiazolidinone derivatives from terephthalic dihydrazide. Eur J Med Chem 2009;44:5112-6.

11. Jain AK, Sharma S, Vaidya A, Ravichandran V, Agrawal RK. 1,3,4-thiadiazole and its derivatives: A review on recent progress in biological activities. Chem Biol Drug Des 2013;81:557-76.

12. Prasad DJ, Ashok M, Karegoudar P, Poojary B, Holla BS, Kumari NS, et al. Synthesis and antimicrobial activities of some new triazolothiadiazoles bearing 4-methylthiobenzyl moiety. Eur J Med Chem 2009;44:551-7.

13. Foroumadi A, Mansouri S, Kiani Z, Rahmani A. Synthesis and in vitro antibacterial evaluation of $\mathrm{N}$-[5-(5-nitro-2-thienyl)-1,3,4-thiadiazole-2yl] piperazinyl quinolones. Eur J Med Chem 2003;38:851-4.

14. Ram VJ, Mishra L, Pandey NH, Kushwaha DS, Pieters LA, Vlietinck AJ. Bis heterocycles as potential chemotherapeutic agents. X. Synthesis of bis (4-arylthiosemicarbazido)-, bis (2-arylamino-1, 3, 4-thiadiazol-5yl) and bis (4-aryl-1, 2, 4-triazolin-3-thione-5-yl) pentanes and related compounds. J Heterocycl Chem 1990;27:351-5.

15. Ergenç N, Ilhan E, Otük G. Synthesis and biological activity of 1,4-disubstituted thiosemicarbazides and their 1,2,4-triazole-5-thione derivatives. Pharmazie 1992;47:59-60.

16. Ram VJ, Pandey HN. Synthesis of 5-membered heterocycles and related compounds. Chem Pharm Bull (Tokyo) 1974;22:2778-83.

17. Reddy KR, Mogilaiah K, Swamy B, Sreenivasulu B. Substituted 1, 8-naphthyridines. Part 11. Synthesis of some 1, 8-naphthyridinylthiosemicarbazides,-triazoles and-thiadiazoles. Cheminformation 1990;21:178.

18. Hiremath SP, Sonar VN, Sekhar KR, Purohit MG. Synthesis of oxadiazolyl-, thiadiazolyl-and triazolylindoles and indolylthiazolidinones. Cheminformation 1989;20:148.

19. Kalyoncuoğlu N, Rollas S, Sür-Altiner D, Yeğenoğlu Y, Anğ O. 1-[p-(Benzoylamino)benzoyl]-4-substituted thiosemicarbazides: Synthesis and antibacterial and antifungal activities. Pharmazie 1992;47:796-7.

20. Sarigol D, Uzgoren-Baran A, Tel BC, Somuncuoglu EI, Kazkayasi I, Ozadali-Sari K, et al. Novel thiazolo[3,2-b]-1,2,4-triazoles derived from naproxen with analgesic/anti-inflammatory properties: Synthesis, biological evaluation and molecular modeling studies. Bioorg Med Chem 2015;23:2518-28.

21. Yehye WA, Abdul Rahman N, Saad O, Ariffin A, Abd Hamid SB, Alhadi AA, et al. Rational design and synthesis of new, high efficiency, multipotent schiff base-1,2,4-triazole antioxidants bearing butylated hydroxytoluene moieties. Molecules 2016;21:847.

22. Sztanke K, Tuzimski T, Rzymowska J, Pasternak K, Kandefer-Szerszeń M. Synthesis, determination of the lipophilicity, 
anticancer and antimicrobial properties of some fused 1,2,4-triazole derivatives. Eur J Med Chem 2008;43:404-19.

23. Haber J. Present status and perspectives on antimycotics with systemic effects. Cas Lek Cesk 2001;140:596-604.

24. Adams WJ, Bombardt PA, Code RA. Absorption of triazolam from pelleted drug-diet mixtures by the mouse: Quantitation of $\alpha$ hydroxytriazolam in urine. J Pharm Sci 1983;72:1185-9.

25. Sidwell RW, Allen LB, Huffman JH, Witkowski JT, Simon LN. Effect of 1- $\beta$-D-ribofuranosyl-1, 2, 4-triazole-3-carboxamide (Ribavirin) on friend leukemia virus infections in mice. Proc Soc Exp Biol Med 1975; 148:854-8.

26. Indiani C, Santoni E, Becucci M, Boffi A, Fukuyama K, Smulevich G. New insight into the peroxidase- hydroxamic acid interaction revealed by the combination of spectroscopic and crystallographic studies. Biochemistry 2003;42:14066-74.

27. Pavlaki M, Zucker S. Matrix metalloproteinase inhibitors (MMPIs): The beginning of phase I or the termination of phase III clinical trials. Cancer Metastasis Rev 2003;22:177-203.

28. Mai A, Massa S, Rotili D, Simeoni S, Ragno R, Botta G, et al. Synthesis and biological properties of novel, uracil-containing histone deacetylase inhibitors. J Med Chem 2006;49:6046-56.

29. Hanessian S, Parthasarathy S, Mauduit M, Payza K. The power of visual imagery in drug design. Isopavines as a new class of morphinomimetics and their human opioid receptor binding activity. J Med Chem 2003;46:34-48.

30. Marks PA. Discovery and development of SAHA as an anticancer agent. Oncogene 2007;26:1351-6.

31. McInnes IB, Schett G. Cytokines in the pathogenesis of rheumatoid arthritis. Nat Rev Immunol 2007;7:429-42.

32. Odake S, Morikawa T, Tsuchiya M, Imamura L, Kobashi K. Inhibition of Helicobacter pylori urease activity by hydroxamic acid derivatives. Biol Pharm Bull 1994;17:1329-32.

33. Whittaker M, Floyd CD, Brown P, Gearing AJ. Design and therapeutic application of matrix metalloproteinase inhibitors. Chem Rev 1999;99:2735-76.

34. Wada CK, Holms JH, Curtin ML, Dai Y, Florjancic AS, Garland RB, et al. Phenoxyphenyl sulfone $\mathrm{N}$-formylhydroxylamines (retrohydroxamates) as potent, selective, orally bioavailable matrix metalloproteinase inhibitors. J Med Chem 2002;45:219-32.

35. Nandy P, Lien EJ, Avramis VI. Inhibition of ribonucleotide reductase by a new class of isoindole derivatives: Drug synergism with cytarabine (Ara-C) and induction of cellular apoptosis. Anticancer Res 1999; 19:1625-33.

36. Kerdesky FA, Schmidt SP, Holms JH, Dyer RD, Carter GW, Brooks DW, et al. Synthesis and 5-lipoxygenase inhibitory activity of 5-hydroperoxy6,8,11,14-eicosatetraenoic acid analogues. J Med Chem 1987;30:1177-86.
37. Cocea E, Grigoras M, Tutoveanu M. Polimeri analogi, nota V: Acizi polihidraxamici si hidrazide copolimere. Bul Inst Polit Iasi 1965;11:159-62.

38. Shiver JW, Fu TM, Chen L, Casimiro DR, Davies ME, Evans RK, et al. Replication-incompetent adenoviral vaccine vector elicits effective anti-immunodeficiency-virus immunity. Nature 2002;415:331-5.

39. McFarland J. The nephelometer: An instrument for estimating the number of bacteria in suspensions used for calculating the opsonic index and for vaccines. JAMA 1907;49:1176-8.

40. Clinical and Laboratory Standards Institute. Performance Standards for Antimicrobial Disk Susceptibility Tests; Approved Standard M2-A9. $9^{\text {th }}$ ed., Vol. 26. Wayne, PA: Clinical and Laboratory Standards Institute; 2006.

41. Bantar C, Schell C, Posse G, Limansky A, Ballerini V, Mobilia L, Comparative time-kill study of doxycycline, tigecycline, sulbactam, and imipenem against several clones of Acinetobacter baumannii. Diagn Microbiol Infect Dis 2008;61:309-14.

42. Kundu S, Abdullah MF, Das A, Basu A, Halder A, Das M, et al. Antifungal ouzo nanoparticles from guar gum propionate. RSC Adv 2016;6:106563-71

43. Bandyopadhyay N, Zhu M, Lu L, Mitra D, Das M, Das P, et al. Synthesis, structure, spectral characterization, electrochemistry and evaluation of antibacterial potentiality of a novel oxime-based palladium(II) compound. Eur J Med Chem 2015;89:59-66.

44. Trott O, Olson AJ. AutoDock vina: Improving the speed and accuracy of docking with a new scoring function, efficient optimization, and multithreading. J Comput Chem 2010;31:455-61.

45. Podust LM, Poulos TL, Waterman MR. Crystal structure of cytochrome P450 14alpha-sterol demethylase (CYP51) from Mycobacterium tuberculosis in complex with azole inhibitors. Proc Natl Acad Sci U S A 2001;98:3068-73.

46. Rose PW, Bi C, Bluhm WF, Christie CH, Dimitropoulos D, Dutta S, et al. The RCSB protein data bank: New resources for research and education. Nucleic Acids Res 2013;41:D475-82.

47. O'Boyle NM, Banck M, James CA, Morley C, Vandermeersch T, Hutchison GR, etal. Open babel:Anopen chemical toolbox. JCheminform 2011;3:33.

48. Reid JR, Heindel ND. Improved syntheses of 5-substituted-4-amino-3mercapto-(4H)-1, 2, 4-triazoles. J Heterocycl Chem 1976;13:925-26.

49. Hase J, Kobashi K, Kawaguchi N, Sakamoto K. Antimicrobial activity of hydroxamic acids. Chem Pharm Bull (Tokyo) 1971;19:363-8.

50. Rossello A, Bertini S, Lapucci A, Macchia M, Martinelli A, Rapposelli S, et al. Synthesis, antifungal activity, and molecular modeling studies of new inverted oxime ethers of oxiconazole. J Med Chem 2002;45:4903-12. 